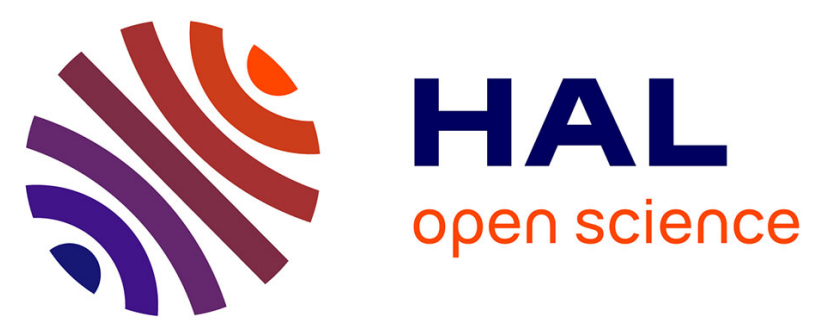

\title{
Presentation, Force Estimation and Control of an instrumented platform dedicated to automated micromanipulation tasks.
}

Micky Rakotondrabe, Cédric Clévy, Kanty Rabenorosoa, Kamel Ncir

\section{To cite this version:}

Micky Rakotondrabe, Cédric Clévy, Kanty Rabenorosoa, Kamel Ncir. Presentation, Force Estimation and Control of an instrumented platform dedicated to automated micromanipulation tasks.. 6th Annual IEEE Confernece on Automation Science and Engineering, CASE'10., Aug 2010, Toronto, Ontario, Canada. pp.722-727. hal-00544883

\section{HAL Id: hal-00544883 \\ https://hal.science/hal-00544883}

Submitted on 9 Dec 2010

HAL is a multi-disciplinary open access archive for the deposit and dissemination of scientific research documents, whether they are published or not. The documents may come from teaching and research institutions in France or abroad, or from public or private research centers.
L'archive ouverte pluridisciplinaire HAL, est destinée au dépôt et à la diffusion de documents scientifiques de niveau recherche, publiés ou non, émanant des établissements d'enseignement et de recherche français ou étrangers, des laboratoires publics ou privés. 


\title{
Presentation, Force Estimation and Control of an Instrumented platform dedicated to Automated Micromanipulation Tasks
}

\author{
Micky Rakotondrabe, Member, IEEE, Cédric Clévy, Member, IEEE, Kanty Rabenorosoa and Kamel NCIR
}

\begin{abstract}
This paper presents a platform used to measure micromanipulation forces. The main interest of the proposed platform is that it uses classical force sensors with limited range and adapts these sensors to that of micromanipulation forces. The proposed platform, and an additional micropositioning device, are afterwards used to develop a micromanipulation system. To automate this system, we design two control levels. First, an internal loop with a PID controller is employed to improve the micropositioning device's performances. Then, an external loop based on an incremental control law is implemented to control the force. The automated system is finally used for the micromanipulation task on rigid and non-rigid microobjects.
\end{abstract}

\section{INTRODUCTION}

The development of tools for micromanipulation and microassembly knows fast progress during the last decade. It is today possible to manipulate a wide variety of microcomponents with sizes comprised between 10 $\mu m$ to $1 \mathrm{~mm}$ with a relative ease. Nevertheless, it is still very difficult to automate such tasks. Developed solutions are very rare and generally correspond to dedicated or specific tasks [1][2].

The lack of automation in the micro-world is mainly due to the specific and numerous difficulties that have to be overcame at the microscale. Especially, automating processes at the microscale requires very small sensors able to measure precisely information the closer possible of the area of interest. Vision based feedback control already shows interesting results but contrary to the macroscale, it is not sufficient in most cases (limitation in the resolution, occulted parts, difficulties to get 2 or $3 \mathrm{D}$ measurements with good refresh rate..). Moreover, surface forces (pull-off, capillary...) are predominant at the microscale and their influence can hardly been detected without adequate force sensors. A previous study shows that pull-off force (i.e. the force to apply to separate two components) can reach $200 \mu \mathrm{m}$ for $50 \times 50 \mu \mathrm{m}^{2}$ planar contacts [3]. Consequently, convenient strategies have to be used and force sensors able to take into account contact forces are required [4].

FEMTO-st Institute,

UMR CNRS-6174 / UFC / ENSMM / UTBM

Automatic Control and Micro-Mechatronic Systems department (AS2M department)

25000 Besançon - France

corresponding author: mrakoton@femto-st.fr
Up to now, few micromanipulators or microassembly stations are based on contact force control [5][6][7]. This is mainly due to the lack of small enough force sensors able to measure forces (in the range of several hundreds of microNewtons) with a good resolution (better than 1 $\mu N)$ and a good enough signal to noise ratio. Recent developments display new and suited measurement devices [8][9][10]. Nevertheless most of them are not applied for the control of micromanipulation or microassembly tasks. This additional step constitutes important difficulties because micromanipulation tasks cause an alternation of open and closed robotic structures that is source of unstabilities. Moreover such a step also requires complex model inversion. Additionally, micromanipulators are generally fragile and only limited compliance can be used to reduce risks to loose gripping components (the presence of surface forces like pull-off prevents from sliding strategies).

To contribute to these lacks, this paper will focus on a force measurement device (design, development and integration in a micromanipulation station) used for the automated micromanipulation tasks. It enables force control in one direction, in the range of $2 m N$ and guarantee a limited compliance (motion $100 \mu \mathrm{m}$ available on the measurement range). This device will notably be extremely helpful to control complex assembly tasks like the assembly of hybrid MOEMS [11][12]. Despite that the developed measurement device in this paper is for 1 degree of freedom (dof) force measurement and estimation, it can be used for multiple dof station with axis decoupling, such as that in [13].

The core of this paper is the presentation and the force estimation on an instrumented platform, its integration into a micromanipulation system and the force control with an end use of automated tasks. In the second section, we present the developed instrumented platform. The third section is dedicated to the characterization and the force estimation on the platform. In the fourth section, we develop a micromanipulation system based on the platform and design control laws in order to perform automated tasks with force control. Experimental results end the paper. 


\section{Presentation of the instrumented PLATEFORM}

Fig. 1-a and b present the principle and the designed instrumented platform respectively. It is based on:

- a table on which micro-objects to be manipulated or pallets for micro-objects are placed,

- four compliant beams (beam-B) which allows a flexure to the table when an external force $F$ is applied to the micro-objects. The four beams are two-end embedded,

- one compliant beam (beam-A) to which a force sensor is connected. This beam is one-end embedded,

- and the base and supports.

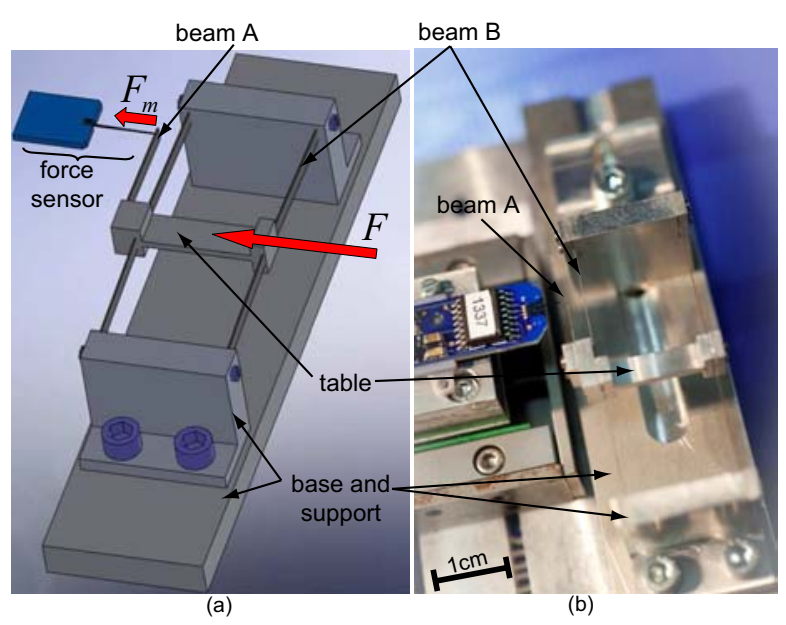

Fig. 1. A picture of the instrumented platform.

When a manipulation force $F$ appears during a micromanipulation or microassembly task, the table moves due to the compliance of the four beams-B. In most of cases, the range of $F$ is inadequate to the measurement ranges of the existing (micro)force sensors. The objective of the beam-A is therefore to adapt the range of the force sensor connected to it and the displacement linked to the specified mechanical compliance. As a result, a force $F_{m}$ much different from the original force $F$ is sensed by the sensor. Beam-A and beam-B are designed accordingly to the used force sensor and to the range of $F$ to be measured. In our case, we use a force sensor FT-S540 from Femtotools [14]) with a range of $0 \rightarrow 170 \mu N$ and a resolution of some tens of nanoNewton. The choice of the Femto-tools force sensor mainly depends on the maximal sizes allowed for the mechanical structure and on the range to be measured.

The objective being the estimation of $F$ using the available information $F_{m}$, the next section is focused on the observer design.

\section{ForCE ESTIMATION}

\section{A. Principle}

Let $F$ be the input and $F_{m}$ be the output of the instrumented platform. So we design in this section an observer in order to reconstitute $F$ using the available information (Fig. 2-a). An intuitive way to trace $F$ is to precisely model the platform and use its inverse model as an observer such as employed in [15] for piezoelectric microactuators. Despite the simplicity of this approach, it requires that the model (which is a transfer function) is inversible, i.e. bicausal and bi-stable. Such a condition is often not respected because most of systems models are strictly causal. To overcome this condition, we propose to use an observer with the structure presented in Fig. 2b, where $\hat{F}$ is the estimate of $F$. In fact this structure has been used to efficiently compensate the creep nonlinearity in piezoelectric microactuators without model inversion [16].

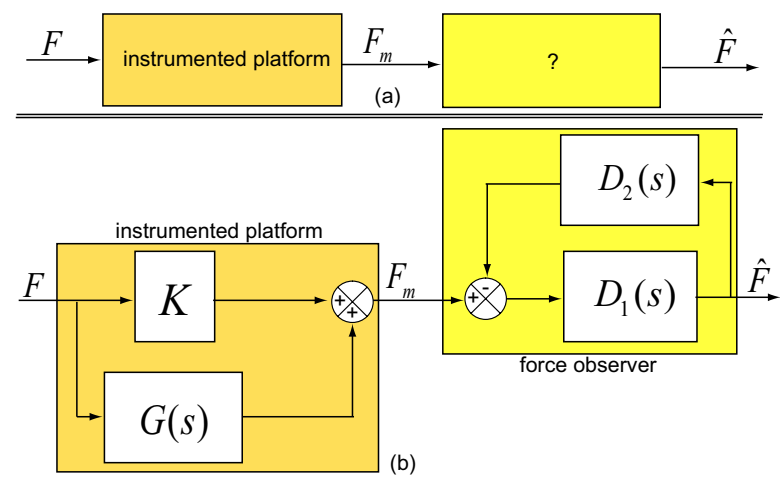

Fig. 2. Bloc diagram of the force observer.

To compute the observer gains $D_{1}(s)$ and $D_{2}(s)$, let us compute the model of the platform(Fig. 2-b):

$$
\frac{F_{m}(s)}{F(s)}=K+G(s)
$$

where $K$ is any strictly positive real and $G(s)$ a transfer function. This model structure is advised when one wants to avoid direct model inversion [16].

Always using Fig. 2-b, the observer transfer function is derived:

$$
\frac{\hat{F}(s)}{F_{m}(s)}=\frac{1}{\frac{1}{D_{1}(s)}+D_{2}(s)}
$$

The objective is that the estimate $\hat{F}$ is equal to the input $F$, i.e $\frac{\hat{F}(s)}{F(s)}=1 \Leftrightarrow \frac{\hat{F}(s)}{F_{m}(s)}=\frac{F(s)}{F_{m}(s)}$. Using the previous condition and (Eq.1) and (Eq.2), we deduct the observer gains:

$$
D_{1}(s)=\frac{1}{K}, \quad D_{2}(s)=G(s)
$$

These results demonstrate that there is no inversion of transfer functions in the observer, and therefore no bi-causality and bi-stability condition on the platform model is required. 


\section{B. Modeling and identification of the platform}

In order to identify the gain $K$ and the transfer $G(s)$, we apply a force $F$ to the plateform and report the measured $F_{m}$. To apply $F$, we use a micropositioning device (the P-611 NanoCube device from PI [17]) with another femtotools force sensor at its extremity. This force sensor, having a range of $0 \rightarrow 2000 \mu N$, serves as end-effector for the micropositioning device and also used to measure $F$ for characterization and identification. Initially, this end-effector and the platform are at their limit contact such as $F=0$. Applying a reference input to the micropositioning device generates a similar input of $F$. The measurement of the different signals and the control of the device are performed using the MATLABSIMULINK (c) software, a computer and a dSPACE-board with a refresh rate of $1 \mathrm{~ms}$.

First, we apply a step input to the micropositioning device. It generates a force constant $F$ to the platform. We report the measured signals $F$ and $F_{m}$. Afterwards, we settle a value of $K$. Then, using the datas $F_{m}$ and $F$, the (Eq.1), the ARMAX method (Auto Regressive and Moving Average eXogenous) and Matlab (C) software, we identify the transfer $G(s)$. Fig. 3 pictures the experimental result and the simulation of the identified model and shows its good accuracy. We have:

$$
\left\{\begin{array}{l}
K=0.001 \\
G(s)=\frac{0.01 \cdot\left(s^{2}-154 s+6.7 \times 10^{5}\right)\left(s^{2}+1958 s+1.9 \times 10^{6}\right)}{\left(s^{2}+167 s+6.7 \times 10^{5}\right)\left(s^{2}-0.27 s+7 \times 10^{5}\right)}
\end{array}\right.
$$

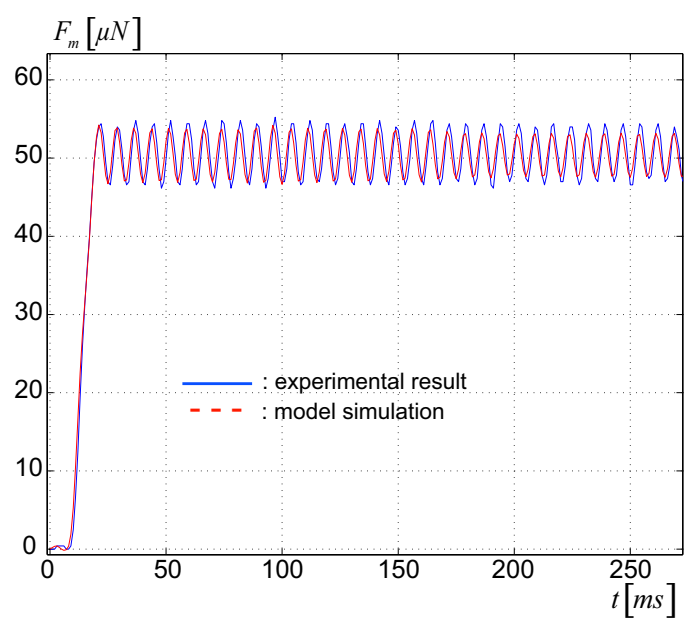

Fig. 3. Step response of the instrumented platform.

In the second experiment, we check the static characteristics of the platform. For that, a sawtooth $F$ signal is applied and the curve $F_{m}$ versus $F$ is plotted. Fig. 4 shows that the system is linear and the previously identified model well coincides to the experimental result. The maximal error - obtained at nearly $45 \mu N$ - is $5 \%$ $\left(=\frac{2.5 \mu N}{45 \mu N}\right)$. It is noticed that if an object with a certain mass is placed on the platform, it could modify the model structure and parameters and therefore the observer. However, since our concern is the manipulation of microobjects - i.e. with negligible mass -, the model uncertainty is negligible.

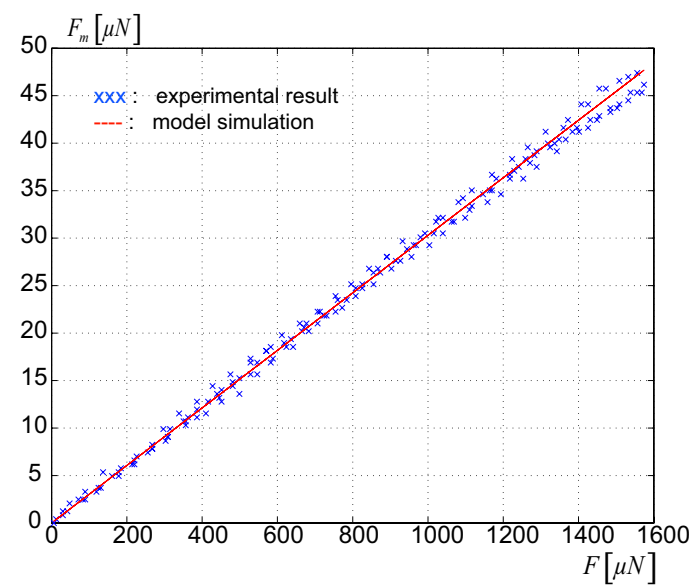

Fig. 4. Static characteristic $\left(F_{m}\right.$ vs $\left.F\right)$ of the instrumented platform.

\section{Implementation of the observer}

Using the observer scheme in Fig. 2-b, the observer gains in (Eq.3) and the identified model as in (Eq.4), the observer was implemented in MatLaB-SimulinK. The refresh rate $(1 \mathrm{~ms})$ was chosen accordingly to the dynamics $G(s)$ which has a bandwith of $1500 \mathrm{rad} / \mathrm{s}$.

The first experiment consists in applying a step input to the previously presented micropositioning device. It generates a constant force $F$ after a certain transient part. This transient part is due to the dynamics of the device and of its end-effector (the second force sensor). Fig. 5 pictures the measurement of $F$ and the estimate $\hat{F}$ and shows that the transient parts of both well coincide.

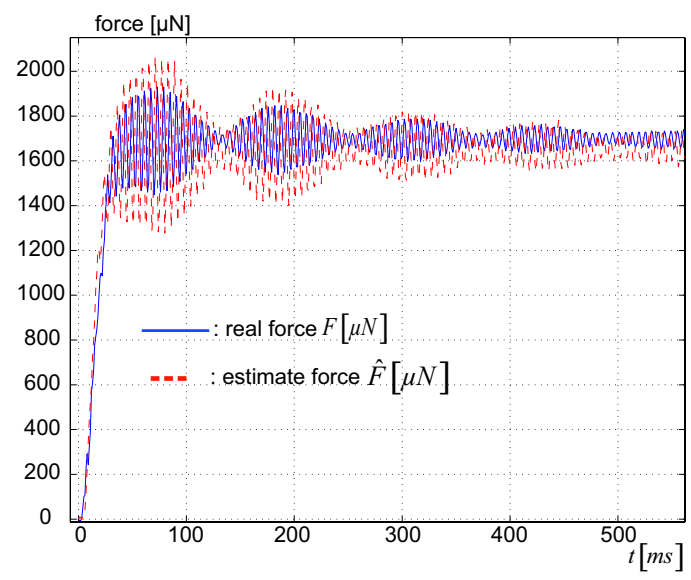

Fig. 5. Transient part of the estimate $\hat{F}$ and of the applied force F

Afterwards, a sawtooth input $F$ is applied to the platform thanks to the micropositioning device and its 
end-effector. Fig. 6 shows the static characteristics of $\hat{F}$ relative to $F$. As $\frac{\partial \hat{F}}{\partial F} \approx 1$, it is deducted that the observer is enough accurate.

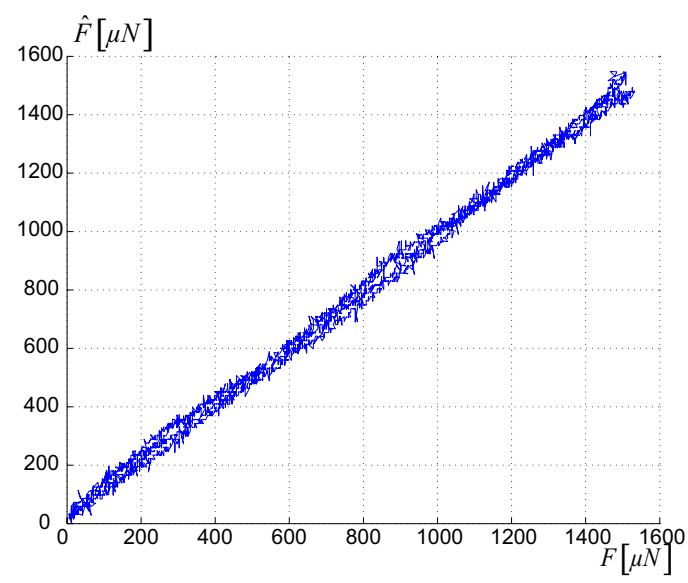

Fig. 6. The estimate $\hat{F}$ vs the applied force $F$.

\section{Application to FORCE CONTROL}

In this section, an application of force control in micromanipulation task is presented. It consists in using a micromanipulator or a microrobot that moves towards the micro-objects until the manipulation force $F$ is equal to a desired value. The micro-objects are placed on the developed platform and the force $F$ is estimated by the previous observer. The proposed application example fits to many tasks in micromanipulation: characterization of the stiffness of biological cells, pick-and-place tasks, friction characterization, etc.

\section{A. Presentation of the complete system}

The previously presented micropositioning NanoCube device with its end-effector (the femtotools force sensor) is used as micromanipulator. This device has a positioning range of $0 \rightarrow 100 \mu \mathrm{m}$ and a resolution of $1 \mathrm{~nm}$. The developed platform and the observer serve as sensor. The experimental setup is pictured in Fig. 7. The steps of the task application is as follows:

- a pallet with micro-objects is placed on the table of the platform,

- the micropositioning device is initially enough far from the platform such as there is no contact between the end-effector and the micro-objects,

- when a reference manipulation force $F_{r}$ is provided by the user, the device approaches towards the micro-objects,

- when a contact appears, the micromanipulator continues to move until $F=F_{r}$. Then, it stops moving.

\section{B. Control principle of the complete system}

Fig. 8 presents a schematic view of the whole system and its force control:

- the estimate force $\hat{F}$ is used as feedback,

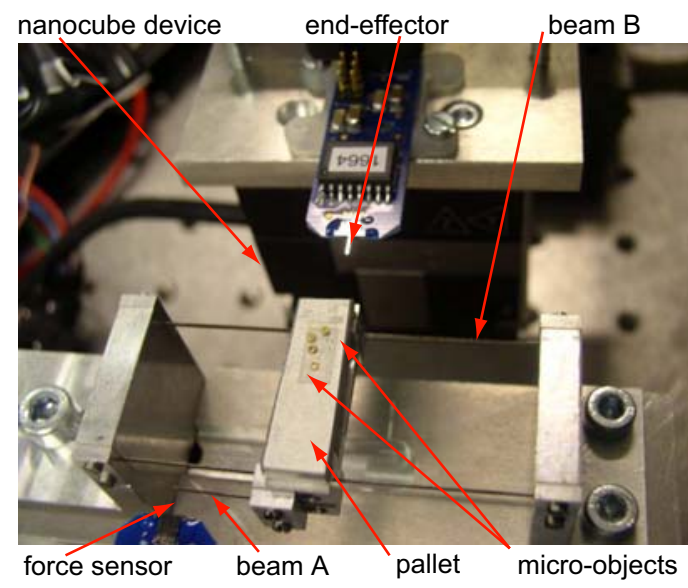

Fig. 7. The experimental setup for the force control.

- the controller $\Sigma$ provides the control signal to the NanoCube device to let it move towards the microobjects until $\hat{F}=F_{r}$,

- to improve the performances of the NanoCube device, it will be controlled using an internal loop controller. The objective mainly concerns the cancellation of the overshots and the diminution of the settling time of the NanoCube. Furthermore, this controller is used to reject the disturbance effect to the NanoCube, especially the reaction force, when the contact between it and the micro-object appears.

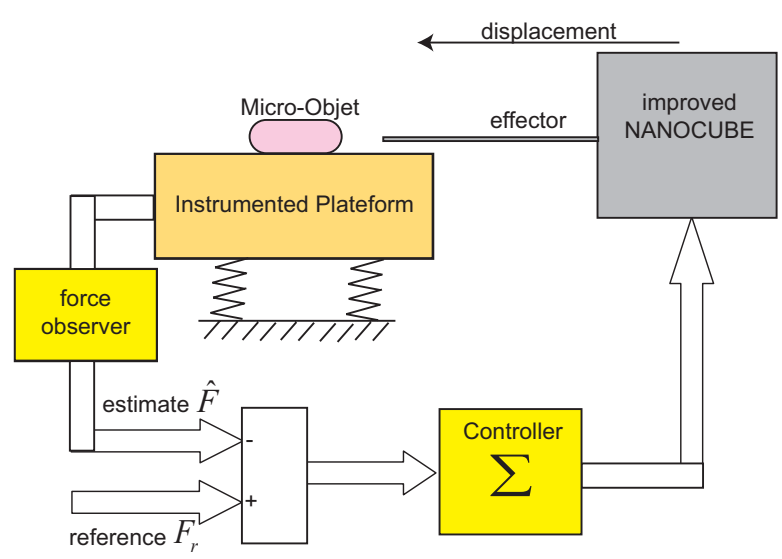

Fig. 8. Principle scheme of the force control of the whole system.

Fig. 9 gives the bloc diagram of the closed-loop control. The internal loop used to improve the performances of the NanoCube is based on a PID controller. We use a Ziegler-Nichols tuning to adjust its parameters. We propose to use an incremental control law with deadzone for $\Sigma$. Indeed, its simplicity and ease of numerical implementation make it very practical in the fields of robotics. We define $\Sigma$ as follows: 


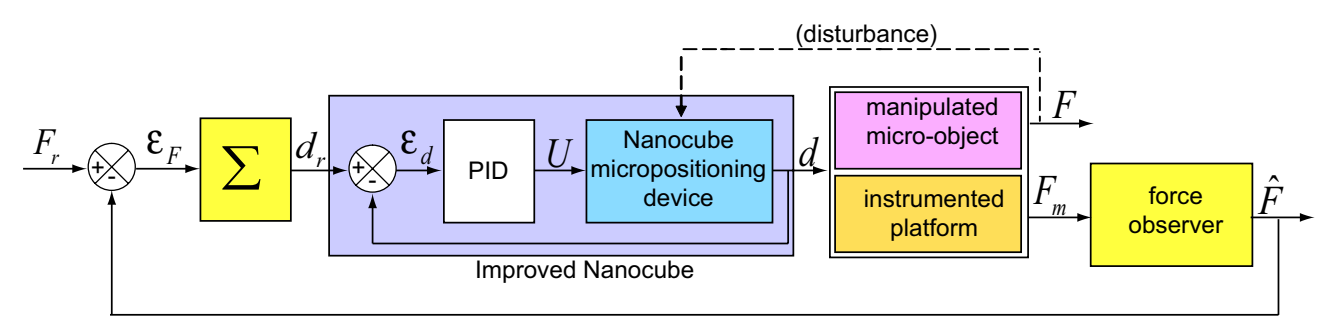

Fig. 9. Bloc diagram of the force control.

$\sum:$

$$
\mid \begin{aligned}
& I F \quad \varepsilon_{F}>\pi_{F} \quad D O \\
& d_{r}(k)=d_{r}(k-1)+d_{\text {incr }} \\
& E L S E I F \quad \varepsilon_{F}<-\pi_{F} \quad D O \\
& d_{r}(k)=d_{r}(k-1)-d_{\text {decr }} \\
& E L S E \\
& d_{r}(k)=d_{r}(k-1) \\
& E N D
\end{aligned}
$$

where:

- $\varepsilon_{F}=F_{r}-\hat{F}$ is the error,

- $\pi_{F}$ indicates an accuracy of measurement which is linked to the noises. We choose $\pi_{F}=2 \mu N$,

- $d_{r}(k)$ and $d_{r}(k-1)$ are the displacement reference for the NanoCube device at time $\left(k T_{s}\right)$ and $\left((k-1) T_{s}\right)$ respectively, with $T_{s}=1 \mathrm{~ms}$ being the refresh rate,

- $d_{\text {incr }}$ and $d_{d e c r}$ are the increment and decrement constants respectively. In our case, we choose $d_{\text {incr }}=$ $d_{\text {decr }}=1 \mathrm{~nm}$. Their values are in general chosen accordingly to the stiffness of the manipulated microobjects. If the micro-object has a very high stiffness, the increment should be very low in order to avoid vibrations at the contact and in order to avoid the destruction of the micro-object and/or of the micromanipulator.

\section{Experimental results}

Our first experiment concerns the manipulation of a rigid micro-object. Fig. 10 shows the experimental results, where $d$ is the displacement of the NanoCube micropositioning device. A reference force $F_{r}=1200 \mu \mathrm{N}$ is applied at time $t=1.5 \mathrm{~s}$. As there is initially nocontact between the end-effector and the micro-object, the NanoCube approaches. At nearly $t=31.5 \mathrm{~s}$, the contact is obtained. The NanoCube continues to move forward while the manipulation force increases until $1200 \mu N$. At time $t=47 \mathrm{~s}$, we set the reference to $F_{r}=0 \mu N$, as a result the NanoCube retracts until the output force is zero.

The speed of the NanoCube, theoretically defined by $V=\frac{d_{i n c r}}{T_{s}}$, can also be experimentally checked through the figure: $\frac{d d}{d t}=1\left[\frac{\mu m}{s}\right]$. We remark that this speed stays unchanged even during the contact, which demonstrates that the PID internal controller well rejects the reaction force (disturbance) applied to the NanoCube. On the other hand, the stiffness of the manipulated micro-object can be characterized from the figure. During the contact, we have:

$$
K_{\text {stiff }}=\frac{d \hat{F}}{d t} \cdot \frac{d t}{d d}=\frac{1200[\mu N]}{1.65[\mu m]}=727\left[\frac{N}{m}\right]
$$
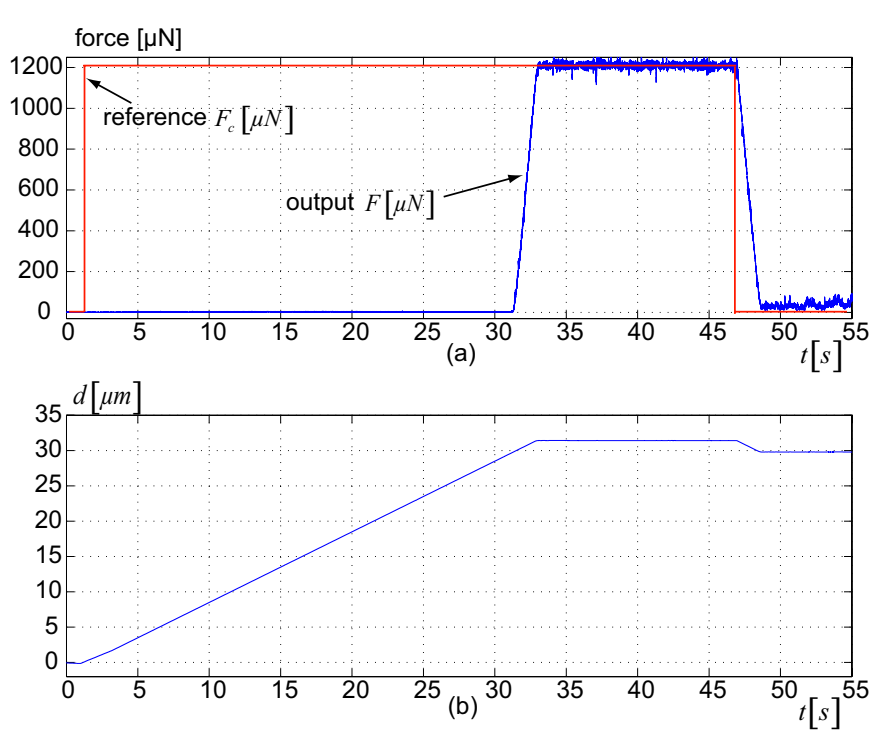

Fig. 10. Force control with a rigid micro-object.

In the second experiment, we use a non-rigid microobject. The same experimental conditions and analysis than with the previous micro-object were used. The results are pictured in Fig. 11. It can be easily checked that the speed of the NanoCube still stays unchanged despite the type of manipulated micro-object. Finally, we characterize the stiffness of the micro-object using the method in (Eq.6), we have:

$$
K_{\text {stiff }}=60\left[\frac{N}{m}\right]
$$

\section{Conclusion}

The objectives of this paper are the presentation of a new force measurement system (instrumented platform), its integration in a micromanipulation system and the control and automation of the whole system.

First, the instrumented platform is presented. Its main function is to adapt the limited range of existing force 

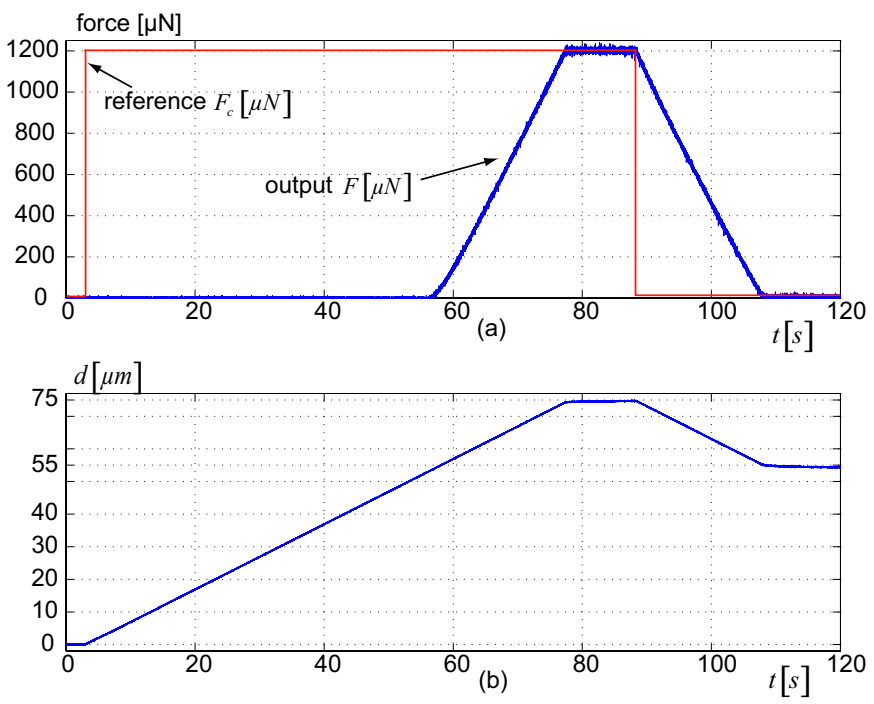

Fig. 11. Force control with a non-rigid micro-object.

sensor to the range necessary in micromanipulation and microassembly contexts. For that, the platform is based on a set of beams and a dynamic observer. The instrumented platform is afterward used in a micromanipulation system and an automated micromanipulation task based on force control is performed. While the platform serves as the feedback measurement, a micropositioning device is used as the actuator. Two levels of control law are proposed. A PID internal loop is first used to improve the performances of the micropositioning device. Then, an external loop is employed to control the force and to automate the task. We use an incremental control law for the external loop because of its simplicity of implementation making it very suited for robotics and therefore microrobotics applications. Finally, tests on the automated micromanipulation system with different objects were performed and show the great interest of the platform in many applications such as objects characterization, microassembly etc.

In future tasks, the developed micromanipulation system will be completed by additional micromanipulators and will be used to perform complete microassembly applications notably for hybrid MOEMS assembly.

\section{ACKNOWLEDGMENT}

This work is supported by the Region FrancheComté and the european FP7SP3-People MicroPAdS project (PIEF-GA-2008-219412). The authors would like to thank David Guibert for his help on the mechanical design.

\section{REFERENCES}

[1] B. Tamadazte, T. Arnould, S. Dembélé, N. Le Fort - Piat and E. Marchand, "Real-time vision-based microassembly of 3D MEMS",IEEE/ASME AIM (International Conference on Advanced Intelligent Mechatronics), 2009.
[2] A. Das, P. Zhang, W. H. Lee, H. Stephanou, and D. Popa, " $\mu^{3}$ : Multiscale, deterministic micro-nano assembly system for construction of on-wafer microrobots", IEEE ICRA (International Conference on Robotics and Automation), pp. 461Ü466, 2007.

[3] K. Rabenorosoa, C. Clévy, P. Lutz, M. Gauthier, and P. Rougeot, "Measurement setup of pull-off force for planar contact at the microscale". Micro Nano Letters, 4, pp. 148 Ü154, 2009.

[4] K. Rabenorosoa, C. Clévy, M. Rakotondrabe and P. Lutz, "Guiding strategies for the assembly of micro-components subjected to planar pull-off force", ASME IDETC-CIE (International Design Engineering Technical Conferences Computers and Information in Engineering Conference), submitted, 2010.

[5] Z. Lu, P. Chen, A. Ganapathy, G. Zhao, J. Nam, G. Yang, E. Burdet, C. Teo, Q. Meng and W. Lin, "A force-feedback control system for microassembly", Journal of micromechanics and microengineering, 2006.

[6] Y. Shen, N. Xi, W. J. Li and Y. Wang, "Dynamic performance enhancement of PVDF force sensor for micromanipulation", IEEE-RSJ IROS (International Conference on Intelligent Robots and Systems), 2005.

[7] S. Khan, A. O. Nergis, A. Sabanovic, and V. Patoglu, "Development of a Micromanipulation System with Force Sensing", IEEE-RSJ IROS (International Conference on Intelligent Robots and Systems), 2007

[8] F. Beyeler, S. Muntwyler, and B.J. Nelson, "Design and Calibration of a Microfabricated 6-Axis Force-Torque Sensor for Microrobotic Applications", IEEE ICRA (International Conference on Robotics and Automation), 2009.

[9] P. Valdastri, S. Roccella, L. Beccai, E. Cattin, A. Menciassi, M. C. Carroza and P. Dario "Characterization of a novel hybrid silicon three-axial force sensor", Sensors and actuators, 2005.

[10] N. Sarkar, A. Geiberger and M. D. Ellis, "Fully released MEMS XYZ flexure stage with integrated capacitive feedback", US Patent, patent nř 6806991B1, 2004.

[11] K. Rabenorosoa, C. Clevy and Lutz, P., "Active force control for robotic microassembly: Application to guiding tasks", IEEE ICRA (International Conference on Robotics and Automation), 2010 .

[12] S Bargiel, K Rabenorosoa, C Clévy, C Gorecki and P Lutz, "Towards microassembly of Hybrid MOEMS Components on Reconfigurable Silicon Free-Space Micro-Optical Bench", Journal of Micromechanics and Microengineering, 2010.

[13] Y. Li and Q. Xu, "Design and analysis of a totally decoupled flexure-based XY parallel micromanipulator", IEEE Transactions on Robotics, vol. 25, no. 3, pp. 645-657, June 2009.

[14] http://www.femtotools.com/

[15] M. Rakotondrabe, Y. Haddab and P. Lutz, "Nonlinear modelling and estimation of force in a piezoelectric cantilever", IEEE/ASME AIM (International Conference on Advanced Intelligent Mechatronics), Zurich Switzerland, Sept 2007.

[16] M. Rakotondrabe, C. Clévy and P. Lutz, "Complete open loop control of hysteretic, creepd and oscillating piezoelectric cantilevers", IEEE Transactions on Automation Science and Engineering, DOI 10.1109/TASE.2009.2028617.

[17] http://www.physikinstrumente.com/ 